\title{
Impaired nonhomologous end-joining in mismatch repair-deficient colon carcinomas
}

\author{
Kwi H Koh ${ }^{1,2}$, Hyun J Kang ${ }^{1,2}$, Long S Li ${ }^{1,2}$, Nam-Gyun Kim ${ }^{1,2}$, Kwon T You ${ }^{1,2}$, Eungi Yang ${ }^{1,2}$, \\ Hyunki Kim ${ }^{1,2}$, Hee J Kim ${ }^{1}$, Chae-Ok Yun ${ }^{2,3}$, Kyung-Sup Kim ${ }^{2,4}$ and Hoguen Kim ${ }^{1,2}$ \\ ${ }^{1}$ Department of Pathology, Yonsei University College of Medicine, Seoul, Korea; ${ }^{2}$ Brain Korea 21 Projects \\ for Medical Science, Yonsei University College of Medicine, Seoul, Korea; ${ }^{3}$ Yonsei Cancer Center, Yonsei \\ University College of Medicine, Seoul, Korea and ${ }^{4}$ Department of Biochemistry and Molecular Biology, \\ Yonsei University College of Medicine, Seoul, Korea
}

\begin{abstract}
Frameshift mutations of coding mononucleotide repeat of the hRAD50 gene and formation of the mutant hMRE11 splicing variant are frequent events in tumors with mismatch repair (MMR) deficiency. Both the hRAD50 and hMRE11 proteins form a heterotrimer with the NBS1, and this heterotrimer is involved in the double strand DNA break repair by homologous recombination and nonhomologous end-joining (NHEJ). In order to clarify the role of $h R A D 50$ and $h M R E 11$ gene alterations in MMR-deficient tumors, we analyzed the expression of the hRAD50 and hMRE11 proteins and we evaluated NHEJ in the seven MMR-deficient and five MMR-proficient colon cancer cell lines. Frameshift mutations of the $h R A D 50$ gene were found in five of seven MMR-deficient cell lines, and this was directly related to the decreased expression of hRAD50 mRNA and protein. The mutant hMRE11 splicing variant was found in all of the seven MMR-deficient cell lines, and this was related to the decreased hMRE11 expression in four of the seven MMR-deficient cell lines. MMR-deficient cell lines with decreased hRAD50 and hMRE11 expressions were more sensitive to $\gamma$-irradiation, and these cell lines showed an impaired NHEJ. The impairment of NHEJ was induced after knockdown of hRAD50 and hMRE11 through small interference RNA. Our findings suggest that mutations of $h R A D 50$ and $h M R E 11$ genes in MMR-deficient tumors are related to the defects in NHEJ, and this may result in chromosomal changes during the progression of tumor.
\end{abstract}

Laboratory Investigation (2005) 85, 1130-1138. doi:10.1038/labinvest.3700315; published online 18 July 2005

Keywords: DNA repair; hMRE11; hRAD50; MMR-deficient; NHEJ

Recent molecular genetic studies have revealed that there is a subset of carcinoma that develops from a deficiency of the DNA mismatch repair (MMR) system. ${ }^{1}$ This genetic pathway, the microsatellite instability (MSI) pathway, begins with the inactivation of one of a group of genes that is responsible for DNA nucleotide MMR, and this leads to extensive mutations in both the repetitive and nonrepetitive DNA sequences with a low frequency of allelic losses, and rare alterations of tumor DNA content have also been noted. ${ }^{2,3}$ The mechanism of tumorigenesis in these high microsatellite instability (MSI-H) tumors is thought to involve the frameshift mutations of microsatellite repeats within the coding regions of the affected

Correspondence: Professor H Kim, MD, PhD, Department of Pathology, Yonsei University College of Medicine, 134 Shinchon-dong, Seodaemun-gu CPO Box 8044, Seoul 120-752, Korea. E-mail: hkyonsei@yumc.yonsei.ac.kr

Received 25 January 2005; revised 17 May 2005; accepted 29 May 2005; published online 18 July 2005 target genes, and the inactivation of these target genes is believed to directly contribute to the development and progression of tumor.

Among the target genes of the MSI-H carcinomas, some of the genes involved in DNA repair are known to be frequently mutated. Frequent frameshift mutations of the coding nucleotide repeat of the hRAD50 gene, and frequent mutations of the microsatellite of the hMRE11 gene and the formation of splicing variants have recently been reported in MMR-deficient tumors. ${ }^{4,5}$ This mutation of the hMRE11 gene leads to the skipping of exon 5, and to the introduction of a premature stop codon in the assembly of a mutant hMRE11 transcript; this mutant hMRE11 transcript thereafter has been referred to as the $484 \mathrm{del} 88$ transcript. Both the hRAD50 and hMRE11 proteins form a heterotrimer with the NBS1 protein, and this heterotrimer is involved in double strand DNA break repair by homologous recombination and nonhomologous end-joining (NHEJ). ${ }^{6}$ The role of this MRN complex in DNA damage signaling and chromosome 
instability had been previously reported on. ${ }^{5,7}$ We have recently found that MMR-deficient tumors with the hRAD50 frameshift mutation have more chromosomal aberrations than those of MMR-deficient tumors without the hRAD50 mutation, and this suggests that chromosomal instability in MSI-H colorectal carcinomas might be induced from the functional alterations of the MRN complex. ${ }^{8}$

In order to clarify the role of hRAD50 and hMRE11 gene alterations in MMR-deficient tumors, we analyzed the relationship between the mutation and the expression status of the MRN complexes, and we measured the DNA repair activities by evaluating NHEJ in the seven MMR-deficient colon cancer cell lines. Our findings suggest that impaired expression of the hRAD50 and hMRE11 proteins is directly related to the mutation status of each gene and the decreased DNA repair activity of NHEJ.

\section{Materials and methods}

\section{Cell Culture and Cell-Free Extracts Preparation}

The 12 cell lines we used were obtained from either the American Type Culture Collection (ATCC; http://www.atcc.org) or the Korean Cell Line Bank (KCLB; http://cellbank.snu.ac.kr). In all, 10 of the colon cell lines, LS174T, SNUC2A, SNUC4, HCT116, Lovo, HCT8, DLD-1, SW480, COLO201 and COLO205 were grown in RPMI medium supplemented with $10 \%$ fetal bovine serum (Life Technologies, Inc., Grand Island, NY, USA), $100 \mathrm{units} / \mathrm{ml}$ penicillin and $100 \mu \mathrm{g} / \mathrm{ml}$ streptomycin at $37^{\circ} \mathrm{C}$ in the presence of $5 \% \mathrm{CO}_{2}$. The other two cell lines, WIDR and HT29, were cultured in Dulbecco's modified Eagle's medium supplemented with $10 \%$ fetal bovine serum (Life Technologies) and antibiotics (100 units/ml penicillin and $100 \mu \mathrm{g} /$ $\mathrm{ml}$ streptomycin). All the cell extracts were prepared by homogenization of cells that were first swollen in hypotonic buffer as described previously. ${ }^{9}$

\section{Screening of MSI}

We used seven (LS174T, SNUC2A, SNUC4, HCT116, Lovo, HCT8 and DLD-1) of the 12 cell lines that were previously reported as being MMR-deficient, and we also used the remaining five cell lines (SW480, HT29, COLO201, COLO205 and WIDR) that were previously reported as being MMR-proficient in terms of their MSI status, as determined by previous studies. ${ }^{10-12}$ The DNAs from these 12 colon cancer cell lines were PCR amplified at three microsatellite loci (BAT25, BAT26 and BAT40) to evaluate the MSI. PCR reactions, separation of PCR products and MSI interpretation were performed as described previously. ${ }^{4,13}$

\section{Detection of Frameshift Mutation}

We analyzed the status of frameshift mutations of the hRAD50 and hMRE11 genes as described previously. . $^{4,13}$ DNA preparation and mutation analysis of the two target genes were performed as described previously. All of the PCR products of these genes showed as one band from the normal DNA, whereas monoallelic or biallelic mutations were detected in some MSI-H tumors (Figures 1 and 2).

\section{Analysis of hRAD50, hMRE11 and NBS1 Expression, and Immunoprecipitation}

For semiquantitative RT-PCR, the RNA $(1 \mu \mathrm{g})$ was reversed transcribed using M-MLV reverse transcriptase (Invitrogen, San Diego, CA, USA) according to the manufacturer's instructions. For each gene, the sequences of the forward and reverse primers were as follows: hRAD50, NM_005732 (Gene Bank accession number), $5^{\prime}$-AACTGCGACTTGCTCCA GAT-3' 5' $^{\prime}$-CAATTATGCTTTGCCTCATGG-3'; hMRE11, NM_005591, 5'-AATATTTTGGAGGAGAATCTTAG GG-3 ${ }^{\prime}, \quad 5^{\prime}$-ATTGAAATGTTGAGGTTGCC- $3^{\prime} ;{ }^{5} \quad \beta$-actin, NM_001101, $5^{\prime}$-TGCTATCCCTGTACGCCTCT-3', $5^{\prime}$ GTACTTGCGCTCAGGAGGAG-3' ${ }^{\prime} \quad \beta$-Actin was used as an internal control. For the Western blot analysis, $20 \mu \mathrm{g}$ of each protein lysate was separated on $6 \%$ SDS-PAGE; this was then blotted onto a polyvinylidene difluoride membrane (Amersham Biosciences) and next probed with the antibodies to hRAD50 (C-19, polyclonal; Santa Cruz, CA, USA and monoclonal $\mathrm{Ab}$ 13B3, Gene Tex. Inc.), hMRE11 (polyclonal; BD Biosciences, Franklin Lakes, NJ, USA), and glyceraldehyde-3-phosphate dehydrogenase (G3PDH; polyclonal; Trevigen, Gaithersburg, MD, USA). The reactions were then developed with ECL-Plus (Amersham Pharmacia Biotech). For immunoprecipitation, the cell lysates $(450 \mu \mathrm{g})$ were precleared and then gently rocked on an orbital shaker with an immunoprecipitating antibody, Nbs1 (Anti-Nibrin/Nbs1; polycolonal; Upstate) at $4^{\circ} \mathrm{C}$. The immune complexes were collected by centrifugation and then boiled to dissociate the immunocomplexes from the beads. The beads were collected by centrifugation and protein separation was performed on the supernatant fraction by SDS-PAGE. To compare the relative binding of hRAD50 and hMRE11 with NBS1, NBS1 was corrected equally.

\section{Cell Survival Assay}

The survival curves for the cell lines were obtained by colony-forming assays. The cells were trypsinized and diluted to form a single-cell suspension that was irradiated (at $0,2,4$ and $6 \mathrm{~Gy}$ ) using a 137 cesium $\gamma$-irradiation source (Gammacell 3000 Elan, MDS Nordion, Ontario, Canada). After irradiation, the cells were diluted so that the appropriate 
MMR-deficient

Mutation status of $h R A D 50+1 / w$

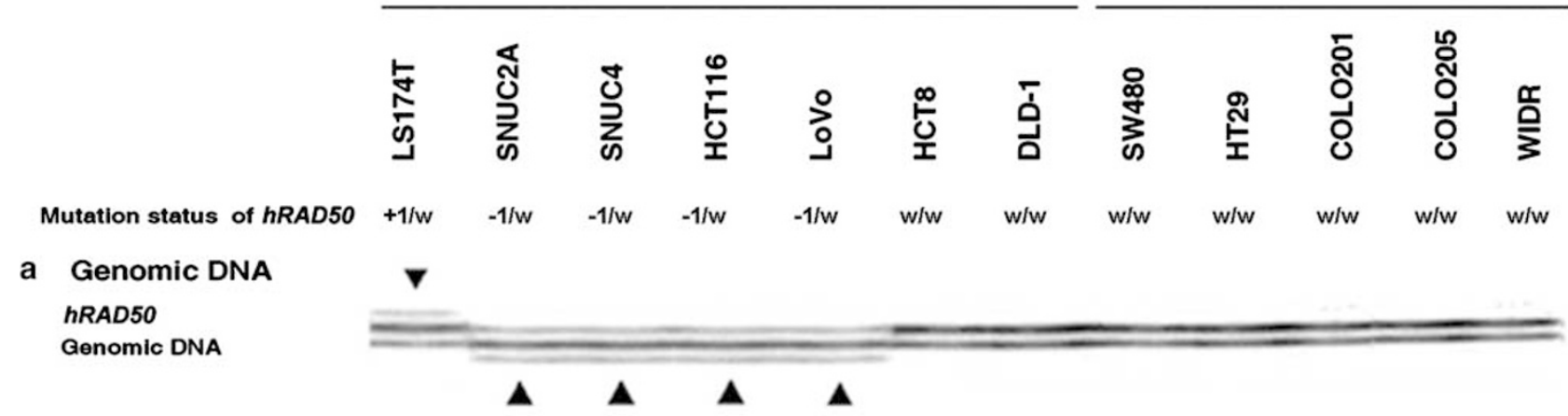

b RNA expression

hRAD50

B-actin

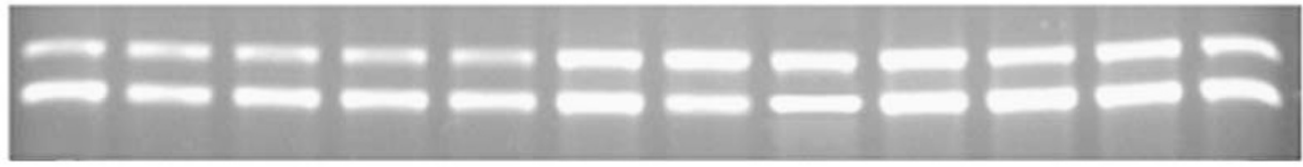

C Protein expression

hRAD50

GAPDH

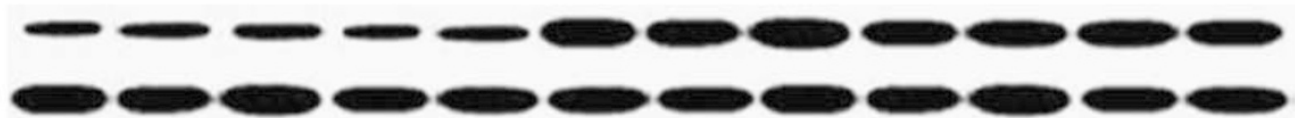

Figure 1 Mutation status of the hRAD50 gene in seven MMR-deficient and five MMR-proficient colorectal carcinoma cell lines (a). Five (LS174T, SNUC2A, SNUC4, HCT116 and Lovo) out of the seven MMR-deficient colon cell lines showed frameshift mutations ( $\boldsymbol{\Lambda}$ ) at the coding nucleotide repeats of the hRAD50 gene. All the mutations were heterozygous; the LS174T cell line showed one base pair insertion and the other four cell lines (SNUC2A, SNUC4, HCT116 and Lovo) showed one base pair deletion. Expression of hRAD50 mRNA and protein in the seven MMR-deficient and five MMR-proficient cell lines (b and $\mathbf{c}$ ). The five MMR-deficient cell lines having the $h R A D 50$ mutation demonstrated the decreased expression of hRAD50 mRNA and protein.

numbers of cells were seeded into $60 \mathrm{~mm}$ culture dishes; the cells were grown for 14 days, then they were fixed and stained with a $70 \%$ ethanol solution and $1 \%$ crystal violet. Colonies containing $>\sim 50$ cells were scored. The percentage of cell survival was determined relative to numbers of colonies from the untreated cells. Each experiment was performed three times for each radiation. The software used for a statistical analysis was the Prism 4.0 (GraphPad Software, San Diego, CA, USA).

\section{Small Interfering RNA Transfection}

hRAD50 and hMRE11 expressions were knocked down by transfection with an siRNA duplex using Silencer $^{\mathrm{TM}}$ siRNA Construction kit (Ambion Inc., Austin, TX, USA). Targeted nucleotides were as follows: hRAD50, 5'-GCGACTTGCT-CCAGATAAA$3^{\prime} ; \quad h M R E 11, \quad 5^{\prime}$-AAATCACTTTGGACGTTCA-3'; Luciferase (as control); 5'-CGTACGCGGAATACTTC GA-3'. Small interfering RNA (siRNA) using lipofectamine $^{\mathrm{TM}} 2000$ (Invitrogen) was transfected to SW480 and HCT116 cells with $100 \mathrm{pmol} / 6 \times 10^{5}$ according to the manufacturer's protocol in six-well plate. To obtain proteins in NHEJ assay of SW480 and HCT116 transfected with siRNA, scales up to $100 \mathrm{~mm}$ culture dishes were performed with the same concentrations of siRNA. All the experiments were carried out $48 \mathrm{~h}$ after transfection, corresponding to $40-50 \%$ confluency of cell lines at the time of siRNA transfection.

\section{Preparation of Substrate DNA and Analysis of NHEJ Activity}

We designed and made the substrate DNA for the end-joining assay that generated a linear dimer as a reaction product without the formation of circular monomers or multimers. First, $2 \mathrm{mg}$ of pBluescript $\mathrm{KS}(+)$ were digested with BamHI and then this was labeled with $\left[\alpha^{32} \mathrm{P}\right] \mathrm{dCTP}$ by a Klenow fill-in reaction using dATP, dTTP, dGTP and $\left[\alpha^{32} \mathrm{P}\right] \mathrm{dCTP}$ for the blunt end formation. After the filling reaction, the Klenow enzyme was inactivated by incubating the reaction mixture at $72^{\circ} \mathrm{C}$ for $15 \mathrm{~min}$. To generate the inert ends that cannot participate in the endjoining reaction, the $5^{\prime}$ phosphate groups of the DNA were removed by a treatment with 10 units of calf intestinal alkaline phosphatase (Promega) for $1 \mathrm{~h}$ at $37^{\circ} \mathrm{C}$. After purification of the treated plasmid DNA 


Mutation status of hMRE11

a Genomic DNA

hMRE11

Genomic DNA

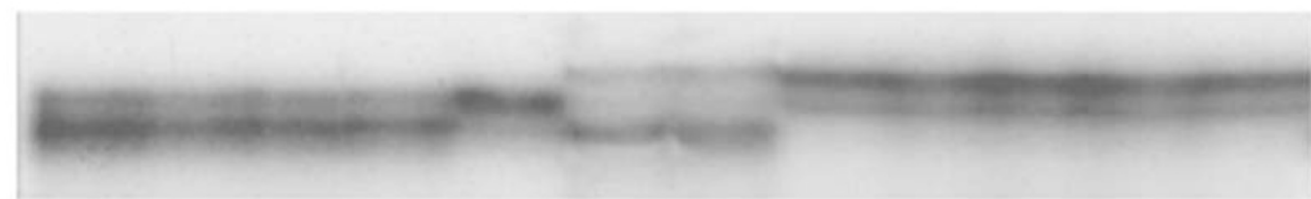

b RNA expression

Wild, Splicing Variant of hMRE11

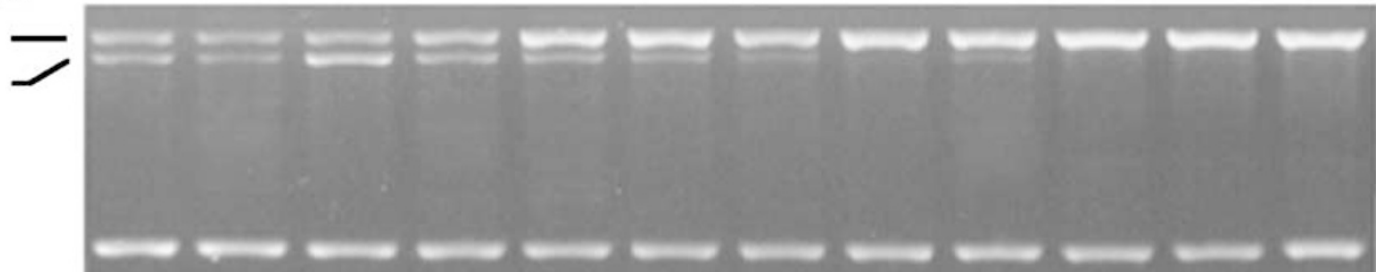

$\beta$-actin

C Protein expression

hMRE11

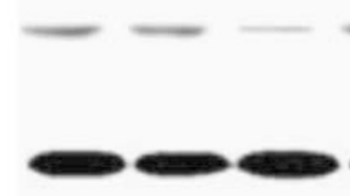

GAPDH

Figure 2 Mutation status of poly T (11) repeats of the hMRE11 gene and the formation of splicing variants of hMRE11 mRNA in the seven MMR-deficient and five MMR-proficient cell lines (a and $\mathbf{b}$ ). All of the seven MMR-deficient cell lines showed frameshift mutations of the poly $\mathrm{T}$ (11) repeat, and no mutation was found in five MMR-proficient cell lines. Spicing variants of the hMRE11 mRNA were detected in all of MMR-deficient cell lines and in one (HT29) MMR-proficient cell lines. Note that four out of seven MMR-deficient cell lines showed the splicing variant mRNA of the hMRE11 gene and the decreased expression of wild-type hMRE11 mRNA. Expression of hMRE11 protein in seven MMR-deficient cell lines and in five MMR-proficient cell lines (c). The protein expression of the hMRE11 gene was correlated to the wild-type mRNA of the hMRE11 gene expression; thus, four cell lines (LS174T, SNUC2A, SNUC4 and HCT116) demonstrated a markedly decreased expression of the hMRE11 protein.

by using a gel extraction kit (Qiagen, Valencia, CA, USA), one of two dephosphorylated ends of the DNA was removed by digestion using EcoRI. The DNA was purified again, and it was then used as a substrate for the final end-joining assay. End-joining assays in the crude cell extracts were carried out as previously described. ${ }^{9}$ The whole-cell extracts were normalized for their respective total protein levels using the Bio-Rad protein assay (Bio-Rad, Richmond, CA, USA). Reactions $(20 \mu \mathrm{l})$ were then carried out in $50 \mathrm{mM}$ triethanolamine- $\mathrm{HCl}(\mathrm{pH} 7.5), 0.5 \mathrm{mM}$ $\mathrm{Mg}(\mathrm{OAc})_{2}, 60 \mathrm{mM}$ potassium acetate, $2 \mathrm{mM}$ ATP, $1 \mathrm{mM}$ DTT and $0.1 \mathrm{mg} / \mathrm{ml}$ BSA. The cell-free extracts were incubated for $5 \mathrm{~min}$ at $37^{\circ} \mathrm{C}$ before the addition of $20 \mathrm{ng}\left[\alpha-{ }^{32} \mathrm{P}\right] \mathrm{dCTP}$ labeled DNA. Positive control joining by T4 ligase (New England Biolabs) was performed using the manufacturer's recommended buffer. The samples were incubated at $37^{\circ} \mathrm{C}$ for $2 \mathrm{~h}$ with the $\left[\alpha{ }^{32} \mathrm{P}\right] \mathrm{dCTP}$ labeled DNA product, and then the samples were deproteinized by proteinase $\mathrm{K}$
$(2 \mathrm{mg} / \mathrm{ml})$ and $0.5 \%(\mathrm{v} / \mathrm{w})$ SDS at $37^{\circ} \mathrm{C}$ for $15 \mathrm{~min}$. The purified DNA substrate was analyzed by electrophoresis on $0.7 \%$ agarose gels, and this was followed by autoradiography. The quantitation of DNA end-joining efficiency was carried out by densitometry.

\section{Results}

Frequent Mutation and the Decreased Expression of hRAD50 in MMR-Deficient Colon Cancer Cell Lines

We found frameshift mutations of the hRAD50 gene in five of the seven MMR-deficient cell lines. No frameshift mutations of the hRAD50 gene were found in the five MMR-proficient cell lines (Table 1). All the mutations were heterozygous; the LS174T cell line showed one base pair insertion, and the other four cell lines (SNUC2A, SNUC4, HCT116 and Lovo) showed one base pair deletion 
Table 1 Microsatellite instability, and mutation status of the coding mononucleotide repeats of the hRAD50, TGF- $\beta$ RII, BAX in 12 colon cancer cell lines

\begin{tabular}{|c|c|c|c|c|c|c|}
\hline \multirow[t]{2}{*}{ Cell lines } & \multirow[t]{2}{*}{ MSI status } & \multirow{2}{*}{$\begin{array}{c}\text { BAT26 } \\
\text { hMSH2 ploy }(A) 26\end{array}$} & \multirow{2}{*}{$\begin{array}{c}\text { MRE11 } \\
\text { poly(T)11 intron } 4\end{array}$} & \multicolumn{3}{|c|}{ Frameshift mutation of CMR in } \\
\hline & & & & hRAD50 & $T G F-\beta R I I$ & $B A X$ \\
\hline LS174T & MSI & + & $-1 /-2$ & $+1 / w$ & $-1 /-1$ & $-1 /-1$ \\
\hline SNUC2A & MSI & + & $-1 /-2$ & $-1 / w$ & $\mathrm{w} / \mathrm{w}$ & $\mathrm{w} / \mathrm{w}$ \\
\hline SNUC4 & MSI & + & $-1 /-2$ & $-1 / w$ & $-1 /-1$ & $-1 / \mathrm{w}$ \\
\hline HCT116 & MSI & + & $-1 /-2$ & $-1 / \mathrm{w}$ & $-1 /-1$ & $-1 / \mathrm{w}$ \\
\hline Lovo & MSI & + & $-1 /-1$ & $-1 / w$ & $-1 /-2$ & $+1 /-1$ \\
\hline НCT8 & MSI & + & $-2 / \mathrm{w}$ & $\mathrm{w} / \mathrm{w}$ & $-1 / \mathrm{w}$ & $\mathrm{w} / \mathrm{w}$ \\
\hline DLD-1 & MSI & + & $-2 / w$ & $\mathrm{w} / \mathrm{w}$ & $-1 / \mathrm{w}$ & $\mathrm{w} / \mathrm{w}$ \\
\hline SW480 & MSS & - & $\mathrm{w} / \mathrm{w}$ & $\mathrm{w} / \mathrm{w}$ & $\mathrm{w} / \mathrm{w}$ & $\mathrm{w} / \mathrm{w}$ \\
\hline HT29 & MSS & - & $\mathrm{w} / \mathrm{w}$ & $\mathrm{w} / \mathrm{w}$ & $\mathrm{w} / \mathrm{w}$ & $w / w$ \\
\hline Colo201 & MSS & - & $\mathrm{w} / \mathrm{w}$ & $\mathrm{w} / \mathrm{w}$ & $\mathrm{w} / \mathrm{w}$ & $\mathrm{w} / \mathrm{w}$ \\
\hline Colo205 & MSS & - & $\mathrm{w} / \mathrm{w}$ & $\mathrm{w} / \mathrm{w}$ & $\mathrm{w} / \mathrm{w}$ & $w / w$ \\
\hline WIDR & MSS & - & $\mathrm{w} / \mathrm{w}$ & $\mathrm{w} / \mathrm{w}$ & $\mathrm{w} / \mathrm{w}$ & $\mathrm{w} / \mathrm{w}$ \\
\hline
\end{tabular}

MSI, microsatellite instability; MSS, microsatellite stability; w, wild type; CMR, the coding mononucleotide repeats.

(Table 1 and Figure 1a). The expression of hRAD50 mRNA was directly related to the mutation status; all of the five MMR-deficient cell lines with the hRAD50 frameshift mutation showed decreased mRNA expression compared to the two MMRdeficient cell lines that were lacking the hRAD50 frameshift mutation, and also compared to the five MMR-proficient cell lines (Figure 1b). The hRAD50 protein expressions, as determined by Western blotting, were directly related to the amount of hRAD50 mRNA expression. The amount of hRAD50 mRNA and protein in these five MMRdeficient cell lines with the hRAD50 frameshift mutation was approximately half to that observed for the other cell lines, as was demonstrated by densitometric analysis.

\section{Formation of the Splicing Variant and the Decreased Expression of hMRE11 in MMR-Deficient Colon Cancer Cell Lines}

We analyzed the status of MSI in the poly-T (11) repeats that are located within intervening sequence 4 (IVS- 4), and they were previously reported to be related to the splicing variant formation. ${ }^{5}$ As we expected, we found frameshift mutations in all of the seven MMR-deficient cell lines, and we found no mutations in the five MMR-proficient cell lines (Table 1 and Figure 2a). We also found the splicing variant form of mRNA for the hMRE11 gene in all of the seven MMR-deficient cell lines and in one MMR-proficient cell line, HT29 (Figure 2b). The amount of wild-type mRNA and protein expression of hMRE11 gene was variable, but cell lines with biallelic mutations showed markedly decreased mRNA and protein expression of hMRE11; four of the 7 MMR-deficient cell lines demonstrated a decreased amount of wild-type mRNA and protein for the hMRE11 gene compared to other three MMR-deficient and five MMR-proficient cell lines (Figure 2c).

\section{MRN Complex Formation is Decreased in the Colon Cancer Cell Lines with the hRAD50 Mutation}

Since the hRAD50 and hMRE11 proteins form a heterotrimer with the NBS1 protein, and the MRN complex is involved in the double strand DNA break repair, we evaluated the amount of the MRN complex by immunoprecipitation (IP). We used the NBS1 antibody for IP, and we evaluated the expression level of hRAD50 and hMRE11 protein compared with the relative amount of NBS1 protein. The amount of MRN complex was markedly decreased in the five colon cancer cell lines with the hRAD50 mutation. These findings indicate that the decreased hRAD50 protein expression of MMR-deficient cell lines with the hRAD50 mutation is the cause of the decreased MRN complex formation (Figure 3).

\section{Sensitivity of Colon Cancer Cell Lines to $\gamma$-Irradiation}

We evaluated the sensitivity of tumor cell lines to $\gamma$ irradiation by using a colony-forming assay (Figure 4). After $\gamma$-irradiation, the five MMR-deficient cell lines displayed worse survival than the three MMRproficient and two MMR-deficient cell lines. At 2 Gy $\gamma$-irradiation, MMR-proficient cell lines had a survival rate greater than $55 \%$, whereas the survival rates of the five MMR-deficient cell lines (LS174T, SNUC2A, SNUC4, HCT116 and Lovo) were below 37\%. Among MMR-deficient cell lines, DLD-1 and HCT8 were less sensitive to $\gamma$-irradiation (their survival was 7.1 and $10 \%$, respectively) compared to a $0 \%$ survival rate for the rest of the five MMRdeficient cell lines at the maximum dose of irradiation, 6Gy. Our data suggest that $\gamma$-irradiation sensitivity is related to the expression status of 


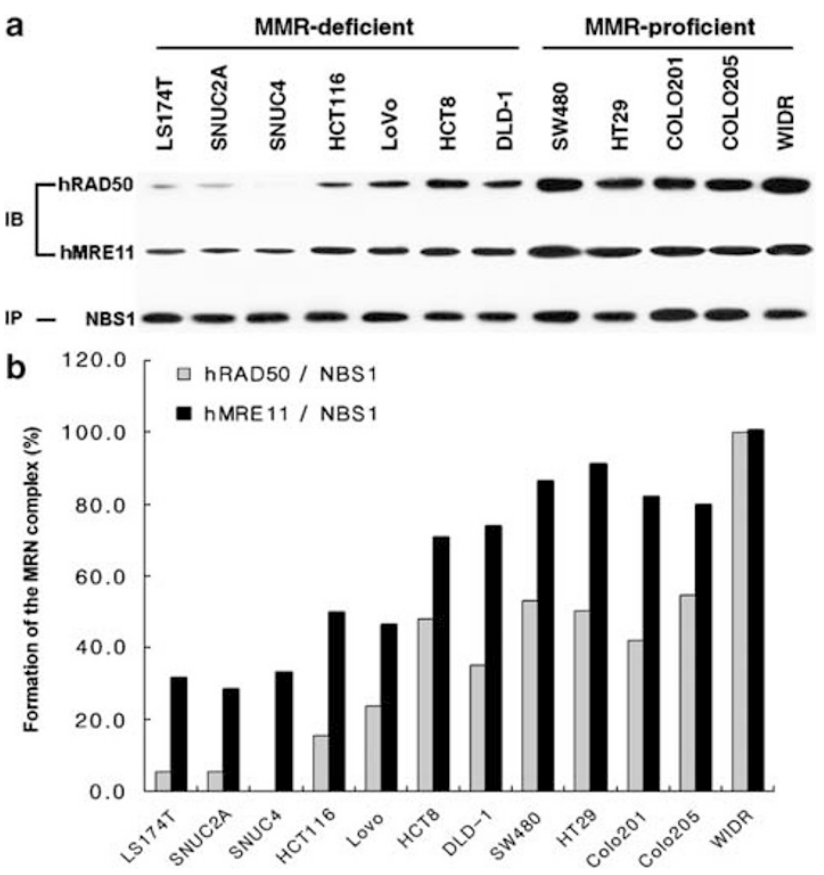

Figure 3 Formation affinity of the MRN complex in the 12 colon cell lines. Nibrin (NBS1) was immunoprecipitated (IP) from $450 \mu \mathrm{g}$ of total proteins per each cell line using a polyclonal antinibrin antibody, and the immunoprecipitates were then separated on $8 \%$ SDS-polyacrylamide gels. The gels were Western-blotted and probed with an anti-nibrin antibody. Blots were stripped and reprobed with an anti-Rad50 antibody and, then with an antihMRE11 antibody (a). Quantitation of RAD50 and hMRE11 protein in individual cell lines relative to total nibrin protein was performed by densitometry (Tina; Raytest Isotopenmeßgeräte GmbH). (b) MRN complex forming affinity of hRAD50 and hMRE11. Binding of NBS1 protein to form hRAD50/NBS1 and hMRE11/NBS1 was lower in the five MMR-deficient cell lines than in the two MMR-deficient and five MMR-proficient cell lines.

hRAD50 and hMRE11 expression. The five MMRdeficient cell lines showed a high sensitivity along with the decreased hRAD50 and hMRE11 expression. In contrast, the remaining two MMR-deficient cell lines (DLD-1 and HCT8) showed normal hRAD50, hMRE11 expression was slightly higher than the five MMR-deficient cell lines, and their sensitivity was not different from the three MMRproficient cell lines.

\section{NHEJ is Decreased in MMR-Deficient Colon Cancer Cell Lines}

We considered the possibility that the impaired expression of the hRAD50 and hMRE11 could result in the abnormal function of the DNA repair mechanism, as related to NHEJ. We measured the end-joining activity in all the 12 human colon cancer cell lines. We used the HeLa cell line as a control for the comparative joining effect. The extracts from MMR-proficient cell lines were more efficient at joining DNA substrates than the extracts

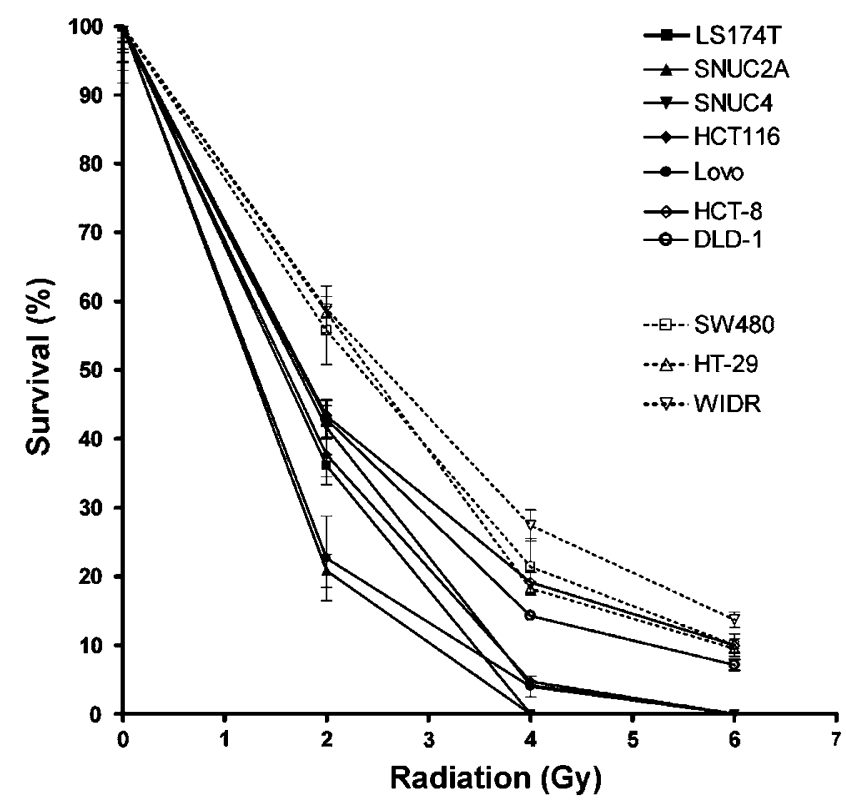

Figure 4 Sensitivity of the tumor cell lines to $\gamma$-irradiation. The cell survival curves for nine colon cell lines were obtained after exposure of $0,2,4$ and $6 \mathrm{~Gy}$ from a $\gamma$-ray source. MMR-proficient cell lines (SW480, HT-29 and WIDR) are shown with the dotted lines and MMR-deficient cell lines (LS174T, SNUC2A, SNUC4, HCT116, Lovo, HCT8 and DLD-1) are shown with the solid lines. MMR-deficient cell lines with decreased hRAD50 and hMRE11 expression showed an increased sensitivity to the $\gamma$-irradiation, more than did the two MMR-deficient cell lines with normal hRAD50 and hMRE11 expression and also more than the three MMR-proficient cell lines.

from MMR-deficient cell lines. When we checked NHEJ effect through the end-joining assay by densitometric analysis in the seven MMR-deficient cell lines, the four cell lines having a decreased hRAD50 expression showed a more decreased NHEJ activity than the two MMR-deficient cell lines having a normal hRAD50 expression (Figure 5). Unlike other four cell lines, one MMR-deficient cell line with decreased hRAD50 expression, HCT116, showed slightly decreased NHEJ activity. To clarify the association between the expression level of the hRAD50 and hMRE11 and NHEJ activity, we performed an siRNA knockdown of the target genes, hRAD50 and hMRE11. siRNA for hRAD50 and hMRE11 was transfected to SW480, a cell line with normal expression of the MRN complex, and HCT116, a cell line with a decreased hRAD50 and hMRE11. Both cell lines showed a knockdown of hRAD50 and hMRE11 after an siRNA transfection study (Figure 6a). NHEJ activity was decreased according to the decreased level of hRAD50 and hMRE11 in both cell lines (Figure 6b). HCT116 with a mutant type of hRAD50 and hMRE11 gene showed a lower end-joining capacity after siRNA treatment than the untreated case. The NHEJ activity of untreated cases of SW480 and HCT116 cells were the same as Figure 5. Our data suggest that the difference of the end-joining effect between 

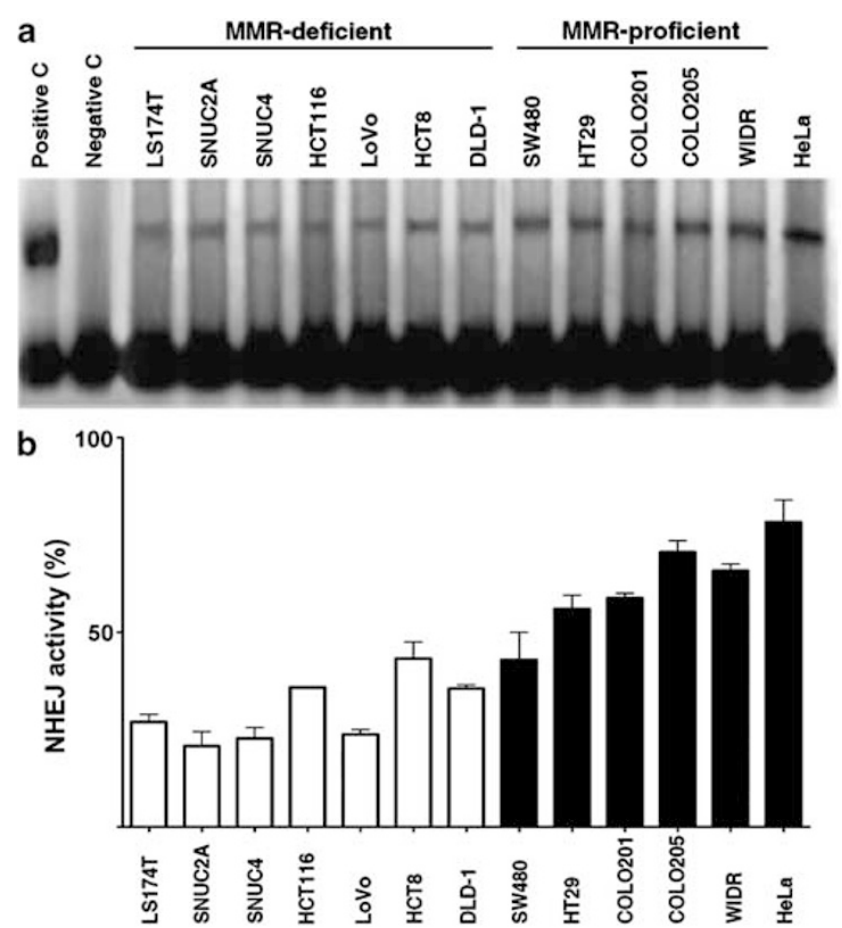

Figure 5 (a) Measurement of NHEJ activities by DNA end-joining assay in the seven MMR-deficient cell lines and the five proficient cell lines. NHEJ activities of MMR-deficient cell lines are decreased more than that of MMR-proficient cell lines. (b) Especially, the four MMR-deficient cell lines (LS174T, SNUC2A, SNUC4 and Lovo) having a decreased hRAD50 expression showed a more decreased NHEJ activity than did the two MMRdeficient cell lines(HCT8, DLD-1) with normal hRAD50 expression by a bar graph. Positive and Negative C is the positive and negative control. The HeLa cell line is used as a control for the comparative end-joining effect.

MMR-deficient cell lines and MMR-proficient cell lines was related to the mutation and the impaired expression status of the $h R A D 50$ and $h M R E 11$ genes.

\section{Discussion}

In this study, we have demonstrated that the expression of hRAD50 and/or hMRE11 is impaired in MMR-deficient cell lines having the $h R A D 50$ and/or hMRE11 mutations. Our finding suggested that there was a relationship between the expression of the hRAD50 and hMRE11 genes in MMR-deficient cell lines and the decreased NHEJ activity. These findings suggest that DNA repair performed through NHEJ system is frequently impaired in MMRdeficient tumors, and this might result in additional chromosomal changes and in the increased sensitivity of MMR-deficient tumors to $\gamma$-irradiation.

Genomic instability is one of the hallmarks of carcinomas. $^{14,15}$ Recent molecular genetic studies have revealed that the molecular genetics of human cancers can be categorized into two major types of genomic instabilities: chromosomal instability (CIN) and MSI. CIN is thought to be caused by continuous losses and gains in the chromosomes during mitosis, and this is characterized by a high frequency of allelic losses, deletions and/or mutations of the tumor suppressor genes. Mutations in the cell-cycle regulators, checkpoint proteins and structural components of the mitotic spindle can be a cause of CIN. ${ }^{16,17}$ The other pathway for genomic instability is the MSI pathway, and it begins with the inactivation of one of a group of genes responsible for DNA nucleotide MMR. The mechanism of tumorigenesis in MSI-H tumors is thought to involve frameshift mutations of microsatellite repeats within the coding regions of the affected target genes, and the inactivation of these target genes is believed to directly contribute to the development and progression of tumor. The reported target genes of MSI-H tumors can be functionally categorized as tumor suppressors and those genes that are involved in apoptosis and DNA repair. ${ }^{18}$ Many genes involved in DNA damage signaling and in the DNA repair pathways play critical roles in the suppression of genome instabilities. We and other researchers have reported observing frequent inactivating mutations of the $h R A D 50$ and $h M R E 11$ genes in MMR-deficient tumors. $^{4,5,19}$ In this study, we confirmed that the mutations of the hRAD50 and hMRE11 genes were frequently observed in MMR-deficient cell lines. The inactivating mutation of the hRAD50 gene and the formation of the inactive hMRE11 splicing variant in MMR-deficient tumors can cause structural chromosomal changes and so result in additional chromosomal instability in MMR-deficient tumors. We have previously reported on the increased chromosome aberrations in MSI-H colon cancers having the hRAD50 mutation. ${ }^{8}$ Therefore, we hypothesized that changes of the DNA repair genes in MMR-deficient tumors might be a link between the CIN and MSI pathways, and so we examined the biological significance of these changes in MMR-deficient tumors.

We suggested an impaired expression of the hRAD50 and/or hMRE11 proteins in MMR-deficient tumors, and this impaired expression is related to the mutation status of the hRAD50 and hMRE11 genes in MMR-deficient tumors. All of MMRdeficient tumors with $h R A D 50$ mutations that we observed were heterozygous. Therefore, the effect of the hRAD50 mutation was a haploinsufficiency because the amount of wild-type hRAD50 mRNA in MMR-deficient tumors with the hRAD50 mutation was approximately half of that compared to the tumors without the hRAD50 mutation. The amount of hMRE11 expression was variable in MMRdeficient tumors. We found monoallelic and biallelic mutations in the poly-T (11) repeat of the hMRE11 gene and in the formation of splicing variants in all of the seven MMR-deficient tumors. By RT-PCR analysis, the amount of the wild-type hMRE11 mRNA and the amount of mRNA from the splicing variants were shown to be variable. For the 
a

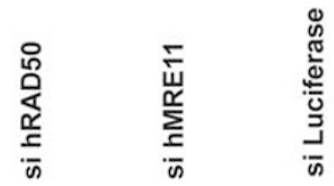
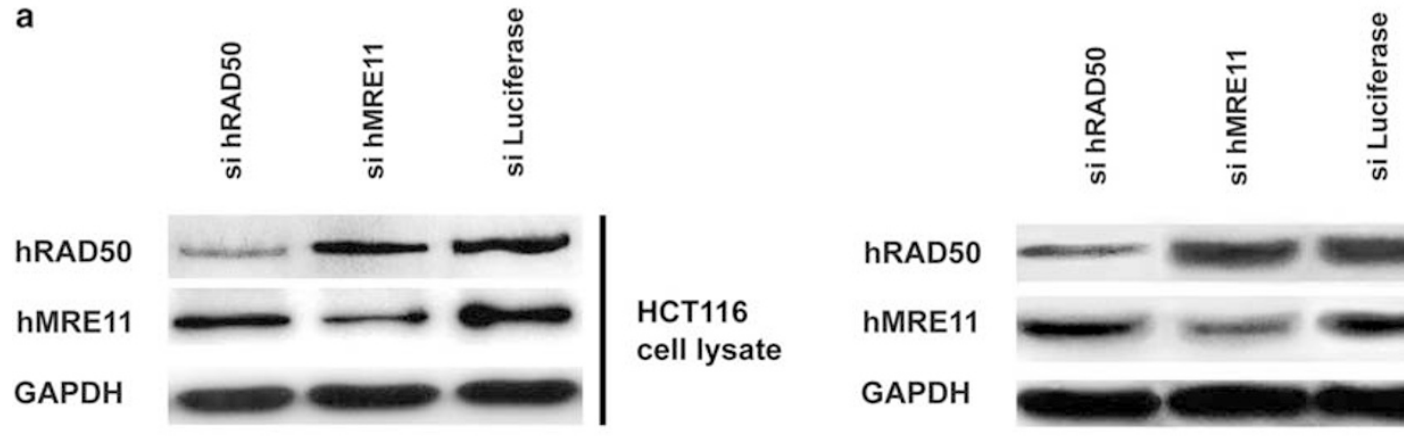

hRAD50

hMRE11

GAPDH

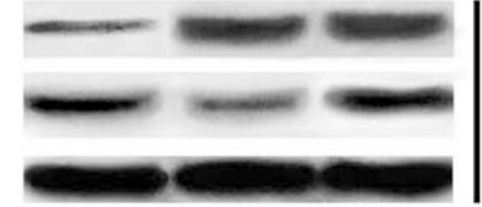

sW480

cell lysate

b

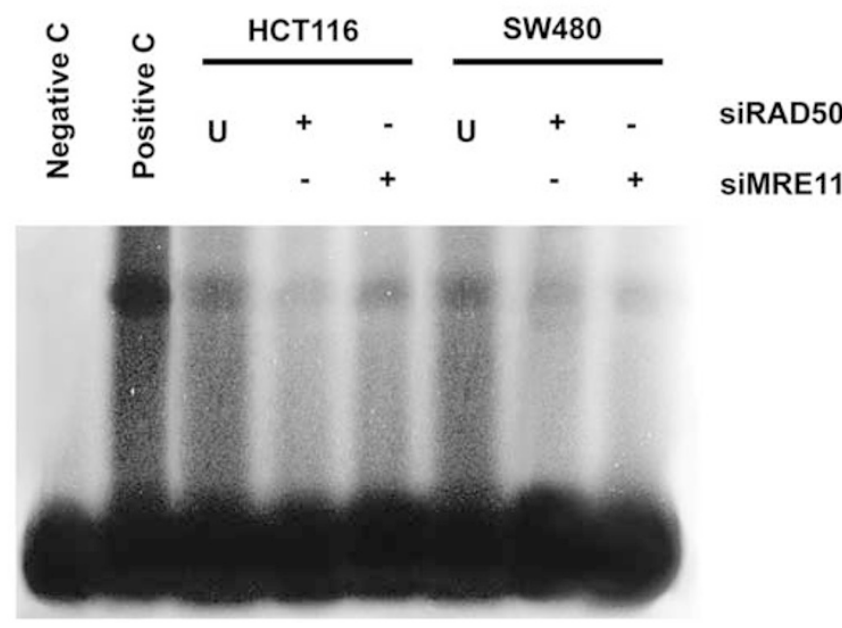

Figure 6 DNA NHEJ activities in HCT116 and SW480 cell lines after a knockdown of hRAD50 and hMRE11 through small interfering RNA (siRNA). SW480 cell line has a wild-type phenotype of hRAD50 and hMRE11, while an HCT116 cell line has a mutant type phenotype of $h R A D 50$ and $h M R E 11$. Immunoblot analysis demonstrated that the target proteins, hRAD50 and hMRE11, were knocked down in both cell lines (a). Decreased end-joining activity was noted in the cell line treated with an siRNA of hRAD50 and hMRE11 (b). $\mathrm{U}$ represents an untreated sample used as a control.

four MMR-deficient cell lines with biallelic mutations, the wild-type mRNA was certainly underexpressed and this was closely related to the markedly decreased expression of the hMRE11 protein. In these cell lines, formation of the MRN complex and its function might be more damaged because these four MMR-deficient cell lines also had hRAD50 and hMRE11 mutations and these cells showed decreased hRAD50 and hMRE11 protein expressions. These impaired expressions of hRAD50 and hMRE11 proteins were expected to result in defective DNA repair and increased genetic instability, and this would lead to somatic mutations in other tumor suppressor genes and in the oncogenes. $^{20}$

We found that the impaired expression of the hRAD50 and/or hMRE11 proteins in MMR-deficient tumors resulted in the decreased MRN complex formation and this was related to the increased sensitivity to $\gamma$-irradiation and the decreased NHEJ. DNA damage signaling and double-stranded DNA break (DSB) repair are included as important parts of a large pool of biological processes that are crucial to maintaining genomic stability. These DSBs are repaired by two main pathways: homologous recombination (HR) and NHEJ. HR is mainly mediated through the replication protein A (RPA), RAD51, RAD52, RAD54, several RAD51 PARALOGUES, the MRN complex and the BRCA proteins. ${ }^{21}$ Proteins involved in NHEJ are the KU heterodimer (KU70, KU80), DNA-dependent protein kinase (DNA-PKcs), DNA ligase IV, XRCC4 and the MRN complex. ${ }^{22}$ The MRN complex has a significant function altogether among the major components involved in the HR and NHEJ pathways, and these pathways are not mutually exclusive. Mutations in the components of the MRN complex result in DNA-damage sensitivity, genomic instability, telomere shortening and aberrant meiosis. In this study, we found higher sensitivity to $\gamma$-irradiation in MMR-deficient cell lines that had decreased hRAD50 and hMRE11 proteins expression. In contrast, the two MMRdeficient cell lines with normal MRN complex formation showed sensitivity that was similar to MMR-proficient cell lines after $\gamma$-irradiation. A similar high sensitivity to the DNA DSB producing 
drug, Bleomycin, had also been reported for MMR-deficient cell lines. ${ }^{23}$ The relationship between the mutation within the components of the MRN complex and a genetic disease is also well known. Hypomorphic mutations of the hMRE11 gene cause an ataxia-telangiectasia-like disease (ATLD), and NBS1 gene mutations cause the Nijmegen breakage syndrome (NBS). A mutation in the RAD50 gene has recently been described in a patient with NBS. ${ }^{24}$ Defects for both DSB pathways might result in enhanced chromosomal aberrations. ${ }^{25}$ A defect of HR repair in MMR-deficient tumor cell lines has been reported. ${ }^{26}$ In this study, we suggest a relationship between impaired NHEJ function and the decreased hRAD50 and/or hMRE11 gene expression. Based on these findings, it can be suggested that mutations of the hRAD50 and hMRE11 genes in MMR-deficient tumors are related to the defects in NHEJ system, and this may result in chromosomal changes during the progression of tumor.

\section{Acknowledgements}

We are grateful to Chang-Hun Lee for his scientific assistance. This study was supported by [FPR05C3460] of 21C Frontier Functional Proteomics Project from Korean Ministry of Science \& Technology and also by a grant from Ministry of Commerce Industry and Energy, Republic of Korea (to C-O Y, MCIE00008131).

\section{References}

1 Lengauer C, Kinzler KW, Vogelstein B. Genetic instabilities in human cancers. Nature 1998;396:643-649.

2 Ionov Y, Peinado MA, Malkhosyan S, et al. Ubiquitous somatic mutations in simple repeated sequences reveal a new mechanism for colonic carcinogenesis. Nature 1993;363:558-561.

3 Thibodeau SN, Bren G, Schaid D. Microsatellite instability in cancer of the proximal colon. Science 1993;260:816-819.

4 Kim NG, Choi YR, Baek MJ, et al. Frameshift mutations at coding mononucleotide repeats of the hRAD50 gene in gastrointestinal carcinomas with microsatellite instability. Cancer Res 2001;6:36-38.

5 Giannini G, Ristori E, Cerignoli F, et al. Human hMRE11 is inactivated in mismatch repair-deficient cancers. EMBO Rep 2002;3:248-254.

6 Petrini JH, Stracker TH. The cellular response to DNA double-strand breaks: defining the sensors and mediators. Trends Cell Biol 2003;13:458-462.

7 Chen C, Kolodner RD. Gross chromosomal rearrangements in Saccharomyces cerevisiae replication and recombination defective mutants. Nat Genet 1999;23: 81-85.
$8 \mathrm{Li}$ LS, Kim NG, Kim SH, et al. Chromosomal imbalances in the colorectal carcinomas with microsatellite instability. Am J Pathol 2003;163:1429-1436.

9 Baumann P, West SC. DNA end-joining catalyzed by human cell-free extracts. Proc Natl Acad Sci USA 1998;95:14066-14070.

10 Lengauer C, Kinzler KW, Vogelstein B. DNA methylation and genetic instability in colorectal cancer cells. Proc Natl Acad Sci USA 1997;94:2545-2550.

11 Park JG, Oie HK, Sugarbaker PH, et al. Characteristics of cell lines established from human colorectal carcinoma. Cancer Res 1987;47:6710-6718.

$12 \mathrm{Ku}$ JL, Yoon KA, Kim DY, et al. Mutations in hMSH6 alone are not sufficient to cause the microsatellite instability in colorectal cancer cell lines. Eur J Cancer 1999;35:1724-1729.

$13 \mathrm{Kim} \mathrm{NG}$, Rhee $\mathrm{H}$, Li LS, et al. Identification of MARCKS, FLJ11383 and TAF1B as putative novel target genes in colorectal carcinomas with microsatellite instability. Oncogene 2002;21:5081-5087.

14 Lengauer C, Kinzler KW, Vogelstein B. Genetic instabilities in human cancers. Nature 1998;396:643-649.

15 Albertson DG, Collins C, McCormick F, et al. Chromosome aberrations in solid tumors. Nat Genet 2003;34: 369-376.

16 Jallepalli PV, Lengauer C. Chromosome segregation and cancer: cutting through the mystery. Nat Rev Cancer 2001;1:109-117.

17 Pihan GA, Purohit A, Wallace J, et al. Centrosome defects and genetic instability in malignant tumors. Cancer Res 1998;58:3974-3985.

18 Perucho M. Tumors with microsatellite instability: many mutations, targets and paradoxes. Oncogene 2003; 22:2223-2225.

19 Vo AT, Zhu F, Wu X, et al. hMRE11 deficiency leads to microsatellite instability and defective DNA mismatch repair. EMBO Rep 2005;6:438-444.

20 Fodde R, Smits R. Cancer biology. A matter of dosage. Science 2002;298:761-763.

21 Zhong Q, Boyer TG, Chen PL, et al. Deficient nonhomologous end-joining activity in cell-free extracts from Brca1-null fibroblasts. Cancer Res 2002;62: 3966-3970.

22 van Gent DC, Hoeijmakers JH, Kanaar R. Chromosomal stability and the DNA double-stranded break connection. Nat Rev Genet 2001;2:196-206.

23 Li HR, Shagisultanova EI, Yamashita K, et al. Hypersensitivity of tumor cell lines with microsatellite instability to DNA double strand break producing chemotherapeutic agent bleomycin. Cancer Res 2004; 64:4760-4767.

24 van den Bosch M, Bree RT, et al. The MRN complex: coordinating and mediating the response to broken chromosomes. EMBO Rep 2003;4:844-849.

25 Takata M, Sasaki MS, Sonoda E, et al. Homologous recombination and non-homologous end-joining pathways of DNA double-strand break repair have overlapping roles in the maintenance of chromosomal integrity in vertebrate cells. EMBO J 1998;17:5497-5508.

26 Mohindra A, Hays LE, Phillips EN, et al. Defects in homologous recombination repair in mismatch-repairdeficient tumour cell lines. Hum Mol Genet 2002;11: 2189-2200. 